Citius, Altius, Fortius- 2019, 12(2), pp. 15-25

\title{
EL DEPORTE, UNA PODEROSA HERRAMIENTA PARA EL ENTENDIMIENTO Y EL DIÁLOGO INTERCULTURAL ${ }^{1}$
}

\author{
Thierry Zintz \\ Universidad Católica de Lovaina (Bélgica) \\ thierry.zintz@uclouvain.be
}

Fecha de recepción: mayo 2019

Fecha de aceptación: septiembre 2019

DOI: $\underline{\text { http://doi.org/10.15366/citius2019.12.2.002 }}$

\section{Resumen:}

Este trabajo se centra, por un lado, en definir el concepto de «Diplomacia deportiva»; pero también en señalar los nuevos actores en invertir y reflexionar sobre el tema. Las ONGs, las instituciones deportivas y las instituciones educativas, son, hoy en día, parte de la diplomacia deportiva blanda en la Unión Europea.

Palabras clave: Diplomacia, deporte, diplomacia deportiva, deporte y diálogo cultural.

\section{Title: SPORT AS AN INFLUENTIAL INSTRUMENT FOR UNDERSTANDING AND INTERCULTURAL DIALOGUE}

\begin{abstract}
:
This definition work highlights in one hand the interest to define this concept "Sport diplomacy" but also the interest of new actors to invest and reflect on the subject. NGO's, but also sport institutions and educational institutions are nowadays part of the soft sport diplomacy in the European Union.
\end{abstract}

Key words: Diplomacy, Sport, Sport diplomacy, Sport and intercultural dialogue.

\section{Perspectiva histórica}

Si bien la relación entre diplomacia y deporte puede parecer obvia e incluso familiar a primera vista, es un objeto de estudio que ha sido poco investigado. (Murray y Pigman, 2014). Varias razones podrían explicar esta situación. Primero, la separación de los campos de investigación dejó estos dos temas en sus respectivas categorías, sin investigarlos ni combinarlos. En segundo lugar, debe entenderse la falta de interés o seriedad que el deporte parecía provocar como tema de estudio hasta los boicots de varios Juegos Olímpicos (Cha, 2009). En tercer lugar, la definición difícil, y en evolución constante, de la relación entre diplomacia y deporte sigue siendo un obstáculo para su correcta comprensión.

Sin embargo, desde finales del siglo $\mathrm{XX} \mathrm{y}$, especialmente desde principios de la primera década del siglo XXI, varias universidades e investigadores han impulsado la reflexión sobre este tema, haciendo de la diplomacia deportiva un objeto de interés y estudio. Es interesante observar que

\footnotetext{
${ }^{1}$ El presente texto, cuyo original fue presentado por su autor en la AOI en inglés, ha sido traducido de forma altruista por Iván Astudillo Villamor, alumno del grado de CCAFyD de la Facultad de Formación de Profesorado y Educación de la Universidad Autónoma de Madrid y Miembro del Comité Juego Limpio del CEO de la UAM.
} 
las reacciones al mismo siguen siendo «esquizofrénicas». De hecho, dependiendo del momento, las circunstancias o las instituciones, el objeto deportivo será considerado importante y serio, a la vez que trivial e insignificante (Jackson, 2013).

El objetivo de esta revisión bibliográfica es contribuir al esfuerzo de revisión a la luz del creciente interés despertado en diversos ámbitos, como, por ejemplo, la Unión Europea en particular; sobre todo, tras la publicación de dos informes de dos Grupos de Alto Nivel sobre diplomacia deportiva, por un lado, y deportes de base, por otro.

Si bien la Unión Europea pretende hacer de la diplomacia y la diplomacia deportiva dos ejes fuertes de su política, tanto extranjera como interna, es importante tener en cuenta ciertas reflexiones e incluso una combinación de conceptos de diplomacia -pública y no gubernamental, dura y blanda, bilateral y multilateral- Diplomacia deportiva y deporte de base.

La Figura 1 pretende presentar estas relaciones antes de proporcionar una visión sobre definiciones y relaciones basadas en una revisión de la literatura científica y técnica.

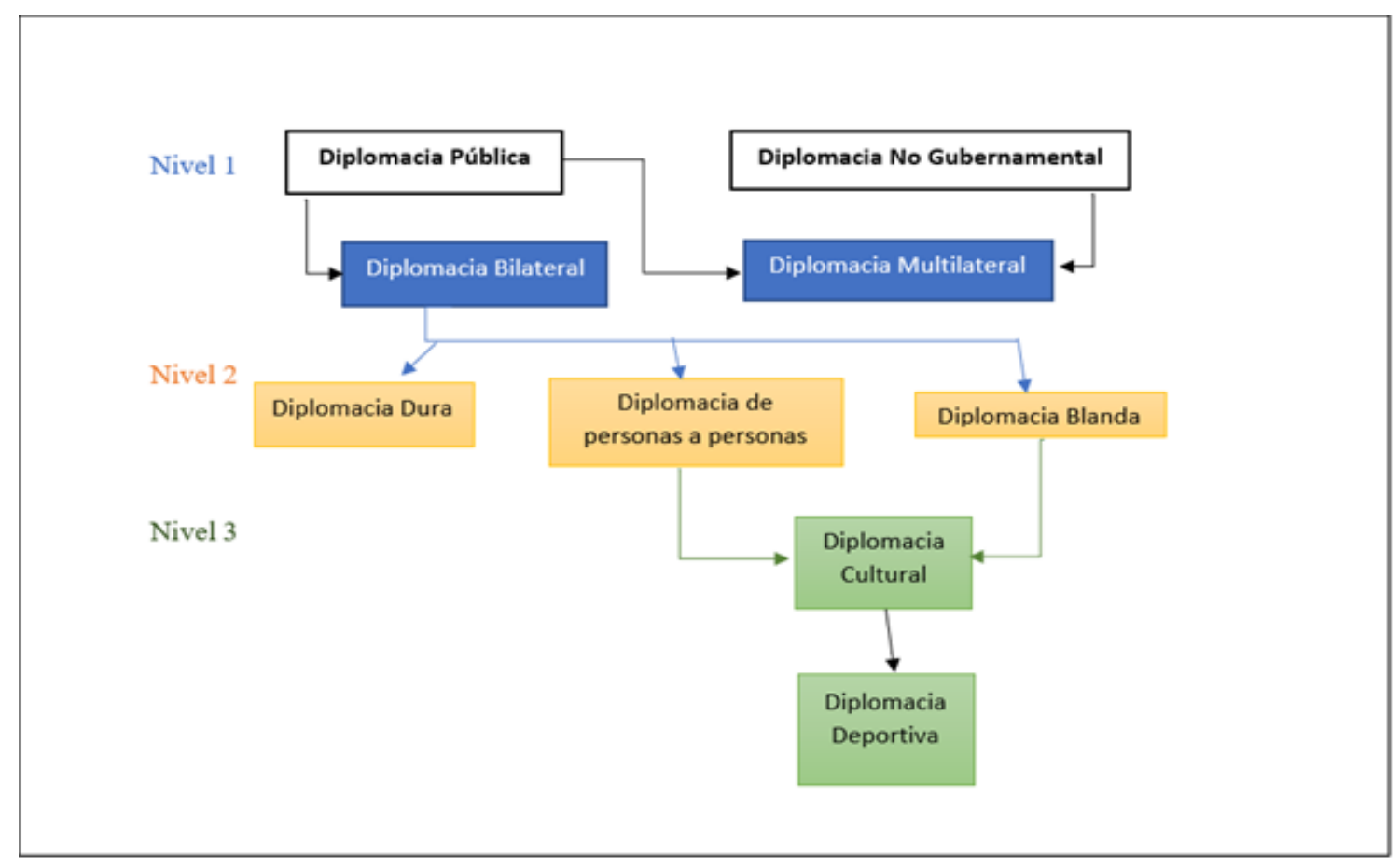

Figura 1. Intento de posicionar la diplomacia deportiva en el contexto general de la diplomacia. 


\section{Conceptualización de la diplomacia deportiva}

\subsection{Diplomacia pública}

No existe un acuerdo unánime que defina la diplomacia pública. Esta falta de consenso puede resultar positiva ya que da lugar a muchas iniciativas.

Veamos unos cuantos rasgos comunes que podríamos destacar:

Es un mecanismo clave a través del cual las naciones fomentan la confianza mutua y las relaciones productivas.

Incluso si sus raíces están muy centradas en el estado, ha evolucionado a la etapa en la que participan una multitud de actores y redes.

Es un claro foco en la promoción del interés nacional y el avance hacia los objetivos de la política exterior de la nación.

Supone un cambio progresivo y constante hacia estrategias de poder suave, como lo define Joseph Nye (es decir, «la capacidad de obtener lo que se desea a través de la atracción en lugar de la coerción o las remuneraciones»).

\subsection{Diplomacia cultural}

La diplomacia pública se refiere principalmente a programas patrocinados por el gobierno destinados a informar o influir en la opinión pública en otros países. Sus principales instrumentos son publicaciones, películas, intercambios culturales de radio y televisión (comunicación unidireccional). Las embajadas y los diplomáticos desempeñan un papel importante en este contexto.

La diplomacia cultural establece una comunicación bidireccional con otros países. El enfoque principal no es meramente político, sino también cultural (deportivo, educativo, artístico). El actor puede asumir su propia agenda independientemente del gobierno. En general, está más centrado en la alta cultura y la educación (menos en la cultura popular, publicaciones, radio o televisión). La diplomacia cultural puede ser patrocinada por el gobierno; pero también por instituciones privadas y ONGs. Las embajadas desempeñan un papel importante, pero no el único.

\subsection{Diplomacia deportiva: ¿qué significa?}

El deporte siempre ha estado y estará vinculado a la política (Beck y Allison, 1993). Así lo ilustra la Tregua Olímpica en la antigua Grecia, la práctica de boicots contra varios países cuyas políticas se denuncian o la instrumentalización de competiciones deportivas por parte de los gobiernos.

Simultáneamente con el desarrollo exponencial del deporte en nuestras sociedades, estamos presenciando la expresión y el surgimiento de un nuevo vínculo entre la política y el deporte a través del reciente desarrollo del concepto «diplomacia deportiva».

La expresión «diplomacia deportiva» es bastante famosa y se ha extendido debido a los diferentes ejemplos que vinculan el deporte y la diplomacia pública. Sin intentar ser exhaustivos, 
podemos enumerar la «diplomacia ping-pong» entre China y los Estados Unidos, la «diplomacia críquet» entre la India y Pakistán, la «diplomacia hockey» entre Canadá y la URSS o incluso la «diplomacia béisbol» entre Cuba y los Estados Unidos. Sin embargo, incluso si se ha discutido la relación entre el deporte y la política, las teorías y prácticas del deporte y la diplomacia están inexploradas en su mayor parte (Murray, 2012). Este hallazgo es extremadamente sorprendente, porque la mezcla de deporte, diplomacia y política es parte del núcleo de las relaciones internacionales. La diplomacia deportiva, un híbrido teórico y práctico de dos instituciones significativas, es la especialización, la explotación y la cosificación de un aspecto familiar de la interacción state-qua-state (Murray, Pigman, 2014).

Como se explica en el informe del Grupo de Alto Nivel sobre diplomacia deportiva, este concepto podría entenderse a través de otros dos conceptos: poder blando y diplomacia pública.

Joseph Nye definió el primero, justo antes del final del colapso de la URSS, como:

«La capacidad de la nación para obtener el resultado deseado no por coerción o pago, sino por atracción, en particular por la atracción de su cultura, sus valores políticos y sus políticas internas y externas» (Nye, 1990, 2004).

La segunda, la diplomacia pública, podría definirse como

«Los mecanismos sin guerra utilizados por un actor internacional (estado, organización internacional, organización no gubernamental, cooperación multinacional u otro actor en el escenario mundial) para gestionar el entorno internacional» (Cull, 2009).

Para Stuart Murray, la diplomacia pública está relacionada con su adaptabilidad. El alcance de la diplomacia pública es ilimitado y el elenco de actores es diverso. Esta diplomacia es significativa: ha creado condiciones fértiles para que surjan formas híbridas de diplomacia (Murray, 2012).

Entre estos dos conceptos, la diplomacia deportiva es tan intuitiva como difícil de definir. Para resolver este problema muchos investigadores han intentado definirla a través de eventos, pero también con la ayuda de conceptos teóricos del deporte por un lado y de la diplomacia por otro.

Por ejemplo, Simon Rofe (2016), al contemplar lo que es nuevo, recoge la opinión de Nicolson sobre la evolución del método diplomático, según escribió en 1953, y considera una «antigua diplomacia» que abarca la antigua diplomacia griega, italiana y francesa. Declaró que estos regímenes deberían considerarse «objetivamente y con cierta realización» como «infinitamente más eficientes» que los métodos de la nueva diplomacia «abierta». Lo fueran o no, la cuestión es que, al estudiar los parámetros del deporte y la diplomacia, hay aspectos valiosos en el pasado y más allá de lo que puede encontrarse a simple vista. Lo que el Nicolson sostiene sugiere que la naturaleza de la diplomacia no cambia: su carácter sí (Rofe, 2016).

Con respecto a la diplomacia deportiva en sí, para James Pamment:

«Es un área vital de investigación que puede desempeñar un papel valioso en la conceptualización de los vínculos entre los distintos conceptos diplomáticos, su impacto mutuo y complementario en el entorno internacional y las modalidades de participación que generan». (Pamment, 2016). 
Si examinamos casos concretos podemos tratar de definir la diplomacia deportiva a través del ejemplo de Sudáfrica. En este caso, Marc Keech consideró que:

«La diplomacia deportiva se ha definido como toda la gama de contactos y competiciones internacionales que tienen implicaciones para las relaciones generales entre las naciones involucradas». (Keech, 2001).

\section{El deporte como instrumento influyente para el entendimiento y el diálogo intercultural.}

Como aclararon Stuart Murray y Geoffrey Allen Pigman, comprender la relación entre deporte internacional y diplomacia juega un papel importante en la comprensión del impacto del deporte internacional en la sociedad en su sentido más amplio (Murray, Pigman, 2013).

Pero, de momento, la mayoría de los artículos encontrados intentan explicar el camino concreto de la diplomacia deportiva y cómo los actores «clásicos» se relacionan entre sí. La mayoría de la representación recae en los estados, aunque algunos actores económicos están cada vez más presentes en la ecuación.

En un artículo de 2001, H.E Chehabi estudió la diplomacia deportiva entre los Estados Unidos e Irán. Mencionó entre muchos otros ejemplos la existencia de otro tipo de diplomacia posible, basada en «relaciones intersociales» $\mathrm{y}$ «diplomacia de persona a persona», por ejemplo, a través del deporte. Su conclusión fue bastante realista, teniendo en cuenta que, si los «contactos deportivos pueden ser útiles en tanto en cuanto pueden hacer que dos países se replanteen sus relaciones, también pueden ayudar a romper el hielo entre las autoridades de ambos lados. Pero los contactos deportivos no pueden por sí mismos conducir a mejores relaciones». (Chehabi, 2001).

Al publicar sus dos informes sobre «Diplomacia deportiva» y «Deporte de base» la Unión Europea pretende mostrar su interés y fe en estos temas. Su combinación de manera práctica y teórica puede llevar a Bruselas a convertirse en pionera en esta área y a alentar a los ciudadanos europeos a participar de manera concreta en estos asuntos.

Además, desarrollar este nuevo concepto complementaría los diferentes tipos de poderes blandos ya implementados por la Unión Europea.

Sin embargo, otros ejemplos del uso del deporte como un instrumento influyente para la comprensión y el diálogo intercultural ya existen, como se explica a continuación.

\subsection{La olimpiada cultural}

La olimpiada cultural era un sueño del Barón Pierre de Coubertin. En sus Memorias olímpicas recordó que:

«No era una simple casualidad que en la antigüedad, los escritores y artistas se reunieran en Olimpia para celebrar los Juegos, creando así el inestimable prestigio que los Juegos han tenido durante tanto tiempo. (...) Sentí que le daría a mi país y al conjunto de la humanidad el estímulo educativo que necesitaban, asi que tuve que restaurar los poderosos contrafuertes que lo habían apoyado en el pasado: el apoyo intelectual, el apoyo moral y en cierta medida el apoyo religioso. A lo que el mundo moderno agregó dos nuevas fuerzas: la mejora técnica y el internacionalismo democrático» (Müller, 2000). 
Este internacionalismo democrático, basado en el deporte y la cultura, representa un instrumento significativo e influyente para el entendimiento y el diálogo intercultural.

\subsection{La educación olímpica como instrumento válido de entendimiento y diálogo intercultural}

Quanz (1993), Binder (2001), Parry (2006), Bronikowski y Bronikowska (2009) han escrito abundantemente sobre los vínculos entre Olimpismo y educación internacional. Según lo sintetizado por Aaron Beacom (2012), el Movimiento Olímpico puede verse como un Movimiento de reforma educativa.

«Si bien la actividad tiene como objetivo influir en el comportamiento y las opciones de vida de los beneficiarios, el proceso educativo también crea un conducto a través del cual se persiguen diplomáticamente los objetivos organizativos. Los programas educativos cada vez más sofisticados, cuyo objetivo aparente es ayudar a lograr su objetivo final de construir un mundo mejor a través del deporte, pueden interpretarse como una 'diplomacia no estatal' clásica, ya que los diversos órganos del Movimiento Olímpico trabajan para contrarrestar los desafios de la legitimidad internacional de este actor global más bien reservado y poderoso». (Beacom, 2012),

La diplomacia del COI hacia las dos Coreas, la «diplomacia Ping Pong» entre China y EEUU, son ejemplos concretos de diplomacia «no estatal» basada en la educación y en un mejor entendimiento.

\subsection{Diálogo de persona a persona entre China y Europa}

El primer diálogo se convocó en 2012 para fortalecer la cooperación en educación, capacitación, cultura, multilingüismo y desarrollo de la juventud.

En 2017, China expresó su intención de fortalecer la cooperación con los países europeos en materia de gestión deportiva y personal.

«Los países europeos tienen una larga tradición de deportes de invierno y Pekín será la sede de los Juegos Olímpicos de Invierno 2022", dijo el ministro Liu. «China pretende inspirar a 300 millones de personas a participar en los deportes de invierno. Esperamos fortalecer la cooperación con los países europeos en la gestión deportiva, así como en la formación e intercambios de atletas, jueces y entrenadores».

Los intercambios de fútbol se introdujeron en 2016 y 3.000 estudiantes, entrenadores y jueces chinos han participado en diversas actividades desde entonces.

Desde entonces China ha enviado a 40.000 estudiantes y académicos a universidades europeas con becas gubernamentales, duplicando el plan original. Al mismo tiempo, unos 5.000 estudiantes europeos han ido a estudiaron en China.

\subsection{La Tregua Olímpica: espejismo o realidad. Diálogo intercultural}

«La presentación y adopción de esta resolución de la ONU cada dos años está lejos de ser el único apoyo que la tregua olímpica recibe de la comunidad internacional antes y durante los Juegos. Las celebridades de todo el mundo también contribuyen a garantizar que los Juegos Olímpicos y el deporte en general ayuden a resolver conflictos y promuevan los valores olímpicos de amistad y respeto. Los principales objetivos perseguidos por el COI a través de la tregua olímpica son movilizar a los jóvenes para la promoción del ideal olímpico, utilizar el deporte para ayudar a construir puentes entre las 
comunidades en conflicto y, en un sentido más amplio, crear una ventana de oportunidad para el diálogo y la reconciliación. Los comités organizadores de los Juegos Olímpicos (OCOG) contribuyen plenamente a promover estos temas, mientras que los compromisos del COI para la tregua olímpica se extienden más allá del período de los Juegos Olímpicos y han llevado a la implementación de una serie de eventos de 'deporte por la paz' a través de sus más de 200 comités olímpicos nacionales». (COI, 2017)

\subsection{Deporte para el desarrollo}

La antigua Oficina de las Naciones Unidas para el Deporte por el Desarrollo y la Paz reconoció el uso del deporte como una herramienta rentable y flexible para promover la paz y los objetivos de desarrollo. En la Declaración de la Agenda 2030 para el Desarrollo Sostenible (Naciones Unidas, 2015) se reconoce aún más el papel del deporte en el progreso social:

«El deporte también es un importante facilitador del desarrollo sostenible. Reconocemos la creciente contribución del deporte a la realización del desarrollo y la paz en su promoción de la tolerancia y el respeto y las contribuciones que hace al empoderamiento de la mujer y de la juventud, los individuos y las comunidades, así como a los objetivos de salud, educación e inclusión social».

\section{El proceso diplomático deportivo europeo}

\subsection{Perspectiva histórica}

Desde hace 20 años, la Unión Europea ha mostrado un creciente interés por el deporte como herramienta. Ya en el Tratado de Ámsterdam de 1997 la Declaración 29 «enfatizaba la importancia social del deporte y, en particular, su papel como catalizador de identidad y del vínculo entre los hombres». En 1999 fue la Declaración de Helsinki sobre el deporte la que pidió a «la Comunidad, sus Estados miembros y el movimiento deportivo reafirmar y fortalecer la función educativa y social del deporte» (Informe de Helsinki sobre el deporte, 1999). Al año siguiente el Consejo Europeo adoptó la declaración sobre las características específicas del deporte y sus funciones sociales en Europa (Niza, 2000). Al mismo tiempo que otras organizaciones están cada vez más interesadas en el deporte como factor de inclusión o como herramienta para la paz, la Comisión Europea está refinando la propia comprensión del uso del deporte que pretende poner en práctica.

Tanto es así, que varias iniciativas se fueron sucediendo, hasta que en 2007 se publicó el Libro Blanco Europeo sobre el Deporte, así como el Plan de Acción de Pierre de Coubertin. Fue un paso importante en la estrategia europea, porque fue la primera iniciativa estratégica integral para abordar temas relacionados con el deporte a nivel europeo. Se trata del resultado de un proceso largo y profundo de consulta entre la Comisión Europea y las partes interesadas del deporte, las autoridades nacionales y los diferentes servicios dentro de la Comisión (Krejza, 2007).

Desde un punto de vista institucional, el artículo 165 del Tratado de Lisboa establece que «la Unión contribuye a la promoción de los desafíos europeos del deporte, teniendo en cuenta sus características específicas, sus estructuras basadas en su función social y educativa voluntaria» (Tratado de Lisboa, 2007) y da muchas posibilidades a la Unión Europea para trabajar en ello y desarrollar su propia estrategia (Murray y Pigman, 2014).

Además, la publicación de los dos informes publicados por los Grupos de Alto Nivel sobre diplomacia deportiva y deportes de base, también son consistentes con esta lógica, que es posible 
gracias a la maduración política y al surgimiento del deporte como una prioridad para la Unión Europea (Garamvölgyi, 2016).

Una referencia directa al uso del deporte para los fines de la diplomacia deportiva europea aparece por primera vez en 2006, cuando la Comisión Europea firmó un «memorando de entendimiento» con la FIFA «para hacer del fútbol una fuerza para el desarrollo en África, en el Caribe y los países del Pacífico»; pero sin añadir ningún detalle sobre la implementación de este acuerdo. Al año siguiente, en 2007, el Libro Blanco de la Comisión sobre el deporte introdujo un uso más general del deporte al subrayar que «el papel social del deporte también tiene el potencial de fortalecer las relaciones externas de la Unión» y que el deporte puede ser una herramienta en la política de desarrollo. En un documento de 2011 denominado «Desarrollo de la dimensión europea del deporte», la Comisión Europea, una vez más, hizo hincapié en que «la cooperación con terceros países europeos, en particular los países candidatos y candidatos potenciales y el Consejo de Europa, debería ser una prioridad». Los dos planes de trabajo de la Unión Europea para el deporte que cubren los períodos 2011-2014 y 2014-2017 no hacen del deporte en las relaciones externas un tema prioritario para el cual se esperen informes por parte de grupos de expertos, aunque ambos piden a la Comisión y a los Estados miembros que:

«Fomenten la cooperación con terceros países, en particular países candidatos y candidatos potenciales para el ingreso en la UE y las organizaciones internacionales competentes en el campo del deporte, incluido el Consejo de Europa» (Husting, 2016).

\subsection{Perspectiva de las políticas}

En su informe al Comisario Navracsics el Grupo de Alto Nivel sobre Diplomacia del Deporte hizo las siguientes declaraciones con respecto a la formulación de políticas de diplomacia deportiva para la UE.

«En un entorno complejo y lleno de desafíos el deporte tiene el potencial de ser una nueva herramienta para:

- Mejorar la política exterior y las relaciones internacionales.

- Alcanzar audiencias externas de manera más profunda, positiva y efectiva.

- Apoyar políticas externas.

- Mejorar la imagen e influencia exteriores.

Existe una coherencia entre los valores del deporte y los objetivos de la política exterior (UE) para preservar la paz, promover la cooperación internacional, desarrollar y consolidar la democracia, el estado de derecho, el respeto de los derechos humanos y las libertades fundamentales.

El deporte resulta ser un lubricante diplomático muy eficaz:

- Crea interés público y buena voluntad pública.

- Apela directamente al público en general.

- Proporciona un entorno favorable para gestionar las relaciones internacionales.

- Facilita cambios y / o aumenta el impulso en las prácticas diplomáticas.

El deporte debe ser un elemento de diálogo y cooperación entre los países miembros y con terceros países como parte de la diplomacia de la UE». (HLG en Sport Diplomacy, 2016) 


\subsection{Buenas prácticas}

En un informe titulado «Diplomacia deportiva: identificación de buenas prácticas» la diplomacia deportiva se describe como:

«Una prioridad clave del Plan de Trabajo de la UE para el Deporte (2017-2020). La diplomacia deportiva es un camino para que la UE y sus Estados miembros refuercen las relaciones diplomáticas, sociales y políticas con países fuera de la UE. El concepto de diplomacia deportiva reconoce el papel que puede desempeñar el deporte para ayudar a la UE a lograr sus diversos objetivos a nivel internacional. También reconoce que el deporte puede mejorar la política exterior nacional e internacional europea, llegar a las partes interesadas externas y mejorar la imagen y la influencia de la UE y sus Estados miembros en todo el mundo. Finalmente, la diplomacia deportiva, al estimular más el diálogo y la cooperación, puede fortalecer los lazos positivos entre los países y buscar mejorar las relaciones más desafiantes entre países».

Este trabajo de definición resalta, por un lado, el interés por definir este concepto, pero también el interés de nuevos actores en invertir y reflexionar sobre el tema. Las ONGs, pero también las instituciones deportivas y las instituciones educativas, son hoy en día parte de la diplomacia deportiva suave en la Unión Europea.

\section{Bibliografía}

- Beacom A (2012), International Diplomacy and the Olympic Movement: The New Mediators. Basingstoke: Palgrave Macmillan. https://doi.org/10.1057/9781137032942

— Beck P., Allison L. (1993), The changing politics of Sport, Manchester : Manchester University Press

- Binder, D. (2001), 'Olympism' Revisited as Context for Global Education: Implications for Physical Education, Quest, 53, pp. 14-34. https://doi.org/10.1080/00336297.2001.10491728

- Bronikowski, M., Bronikowska, M. (2009), Can Olympic Education be among the Peacemakers, in Georgiadis, K., and Syrigos, A. (eds) (2009), Olympic Truce as a Platform for Peace, Athens, The International Truce Centre, pp. 93-109.

- Cha, V. D. (2009), Beyond the Final Score: The Politics of Sport in Asia, New York: Columbia University Press. https://doi.org/10.7312/cha-15490

- Chehabi, H. E. (2001). Sport Diplomacy between the United States and Iran. Diplomacy \& Statecraft 12 (I, March): 89 - 106. https://doi.org/10.1080/09592290108406190

— Cull, N. J. (2009). Public Diplomacy: Lessons from the Past. Los Angeles: Figueroa Press.

— De Groot, GJ. (2011), Sport and Politics - Sometimes a Good Mix, ISN Insights.

- Jackson, S.-J. (2013), The contested terrain of sport diplomacy in a globalizing world. https://doi.org/10.1177/2233865913498867 
- Fourneyron, V., Zintz, T., (Eds.) Report to Commissioner Tibor Navracsis, High level group on Sport diplomacy, 29/06/2016,

- http://ec.europa.eu/assets/eac/sport/policy/cooperation/documents/290616-hlg-gs-finalreport en.pdf, recuperado el 21/03/2019.

— Garamvölgyi B. (2016), Sport: a tool for reaching EU external policy goals?

- Husting Alexandre (2016), Unpublished reference document for the High Level Group on Sport Diplomacy.

- Keech M., (2001), The Ties that Bind: South Africa and Sport diplomacy 1958-1963, The Sport Historian 21 no.1, 71-93. https://doi.org/10.1080/17460260109443377

- Krejza, M. (2007), The European Commission's White Paper on Sport, International Sports Law Journal (3) 4, 73-76.

- Manzenreiter, W. (2008), Football diplomacy, post-colonialism and Japan's quest for normal state status, Sport in Society: Cultures, Commerce, Media, Politics, 11:4, 414-428. https://doi.org/10.1080/17430430802019359

- Müller, N. (2000), Pierre de Coubertin, Olympism - Selected Writings, International Olympic Committee, pp. 620-621.

- Murray S. (2012). The Two Halves of Sports-Diplomacy, Diplomacy \& Statecraft, Volume 23, Issue 3, pp 576-592. https://doi.org/10.1080/09592296.2012.706544

- Nye J. (1990), Soft Power. Foreign Policy n80, Autumn 1990, pp 153-171. https://doi.org/10.2307/1148580

— Nye J. (2004), Soft power: The means to success in world politics. New York: Public Affairs.

- Pamment J. (2016), Rethinking Diplomatic and Development Outcomes through Sport: Toward a Participatory Paradigm of Multi-Stakeholder Diplomacy, Diplomacy \& Statecraft, 27:2, 231250. https://doi.org/10.1080/09592296.2016.1169787

- Parry, J. (2006), Sport and Olympism: Universals and Multiculturalism, Journal of the Philosophy of Sport, 33, pp. 188-204. https://doi.org/10.1080/00948705.2006.9714701

- Pigman G. \& Rofe J.S (2014) Sport and diplomacy: an introduction, Sport in Society: Cultures, Commerce, Media, Politics, 17:9, 1095-1097.

- Quanz, A. (1993) Civic Pacifism and Sports-Based Internationalism: Framework for the Founding of the International Olympic Committee, The International Journal of Olympic Studies, 2, pp. 1-23.

- Rofe J.S (2016), Sport and diplomacy: a global diplomacy framework, Diplomacy \& Statecraft, Volume 27, pp212-230. https://doi.org/10.1080/09592296.2016.1169785 
- S.N., "Sport Diplomacy: identifying good practices". A final report to the European Commission, January 2018 - Written by Ecorys.

- S.N., The Helsinki Report on Sport, Report from the Commission to the European Council with a view to safeguarding current sports structures and maintaining the social function of sport within the Community framework, 1999.

- S.N., The Olympic Truce, https://www.olympic.org/news/the-history-of-the-olympic-truce , recuperado el 21/03/2019.

- S.N., The United Nations, 2030 Agenda for Sustainable Development, https://www.un.org/sustainabledevelopment/development-agenda/, recuperado el 21/03/2019. 